\title{
DIFFRACTION PROFILE POLE FIGURES MEASURED WITH A POSITION SENSITIVE DETECTOR
}

\author{
L. WCISLAK and H. J. BUNGE \\ Department of Physical Metallurgy, Technical University of Clausthal, \\ D-38678 Clausthal-Zellerfeld, Germany
}

(Received 16 February 1996)

Pole figures in the classical sense are defined by the integral intensities of Bragg reflections. The conventional technique of pole figure measurement uses a single detector (usually a scintillation counter) with a wide receiving slit where the integral intensity of a given Bragg reflection is obtained directly. The usage of a position sensitive detector instead of a single detector allows to measure whole diffraction profiles simultaneously. Integral intensities of the diffraction peaks can then be obtained mathematically using peak profile analysis both on overlapped (profile deconvolution) and separated (profile fitting) reflections.

The peak profile analysis provides, besides the integral intensity, also several other peak parameters characterizing an individual (hkl)-reflection. These are the peak maximum intensity, peak position or peak shift, peak width or peak shape parameters, the local peak background, as well as the fitting error. The availability of all these peak profile parameters in each sample position allows to define and plot new generalized "pole figures". Besides the texture information contained in the integral intensity pole figures these generalized pole figures contain information about different other microstructural quantities, e.g. particle size, lattice strain, diffuse scattering and amorphous phases. Fitting error pole figures can be interpreted as a measure of the experimental accuracy and profile fitting effectivity.

Some examples of conventional integral intensity pole figures and generalized diffraction profile pole figures in metal and polymer samples are presented.

KEY WORDS: X-ray polycrystal diffraction, position sensitive detector, detection homogeneity, peak profile analysis, integral intensity pole figure, generalized diffraction profile pole figures

\section{INTRODUCTION}

The majority of all experimental texture studies is carried out by means of polycrystal diffraction and is based on the measurement of pole density distributions (pole figures), followed by the calculation of the orientation distribution function ODF of crystallites (Bunge, 1982). Pole figures are defined by the integral intensities of Bragg reflections (hkl) depending on the orientation of the diffraction vector relative to the sample coordinate system. X-ray diffraction pole figure measurements are mostly based on the angular dispersive method using monochromatic radiation in which several pole figures are distinguished by their respective diffraction angles $\theta_{\text {hkl }}$ which are related to the reflecting lattice planes (hkl) by the Bragg equation. Reflection takes place in all those crystallites for which the diffraction condition, Eq.(1), is fulfilled

$$
\overrightarrow{\mathrm{h}} \| \overrightarrow{\mathrm{S}} \perp \text { (hkl) }
$$


where $\vec{h}$ is the crystal direction perpendicular to the lattice plane (hkl) and $\vec{S}$ is the diffraction vector fixed by the setting of incident and reflected beam direction. All sample directions $\overrightarrow{\mathrm{y}}=\{\alpha \beta\}$ are then successively brought into the diffraction direction $\overrightarrow{\mathrm{S}}$ in order to measure their particular diffracted intensities. If the sample is oriented in such a way that its direction $\vec{y}$ is parallel to the diffraction vector $\vec{S}$

$$
\overrightarrow{\mathrm{y}} \| \overrightarrow{\mathrm{S}}
$$

then the reflected intensity $\mathrm{I}_{(\mathrm{hkl})}(\alpha \beta)$ is proportional to the volume fraction of crystallites being in the reflection position i.e. to the (hkl)-pole density $\mathrm{P}_{(\mathrm{hkl})}(\alpha \beta)$

$$
\mathrm{I}_{(\mathrm{hkl})}(\alpha \beta)=\mathrm{N}_{(\mathrm{hkl})} \cdot \mathrm{P}_{(\mathrm{hkl})}(\alpha \beta)
$$

where $\mathrm{N}_{(\mathrm{hkl})}$ is the normalization factor depending on the intensity of the incident beam, the irradiated sample volume, the reflectivity of the given lattice plane (hkl) and on some other factors. The above equation is the basis of experimental texture analysis (Bunge, 1986).

Texture measurements in polycrystalline samples are carried out using a texture diffractometer which mostly uses Bragg-Brentano focusing geometry and a flat sample as is shown in Figure 1 (see e.g. Bunge and Puch, 1984). The sample is mounted with its surface in the axis of the diffractometer circle whereas the X-ray source and the radiation detector can move along this circle. The primary beam is diffracted within a thin layer of the compact sample given by the penetration depth of the X-rays in the sample. The lateral extension of the illuminated spot on the sample surface is determined by the divergence of the incident beam. If this extension is small the surface of the sample can be considered to be a part of the focusing circle, see Figure 1 . All $\mathrm{X}$-rays emitted at one point of the focusing circle are thus focused into one other point on this circle where the detector is positioned. In practice, however, deviations from
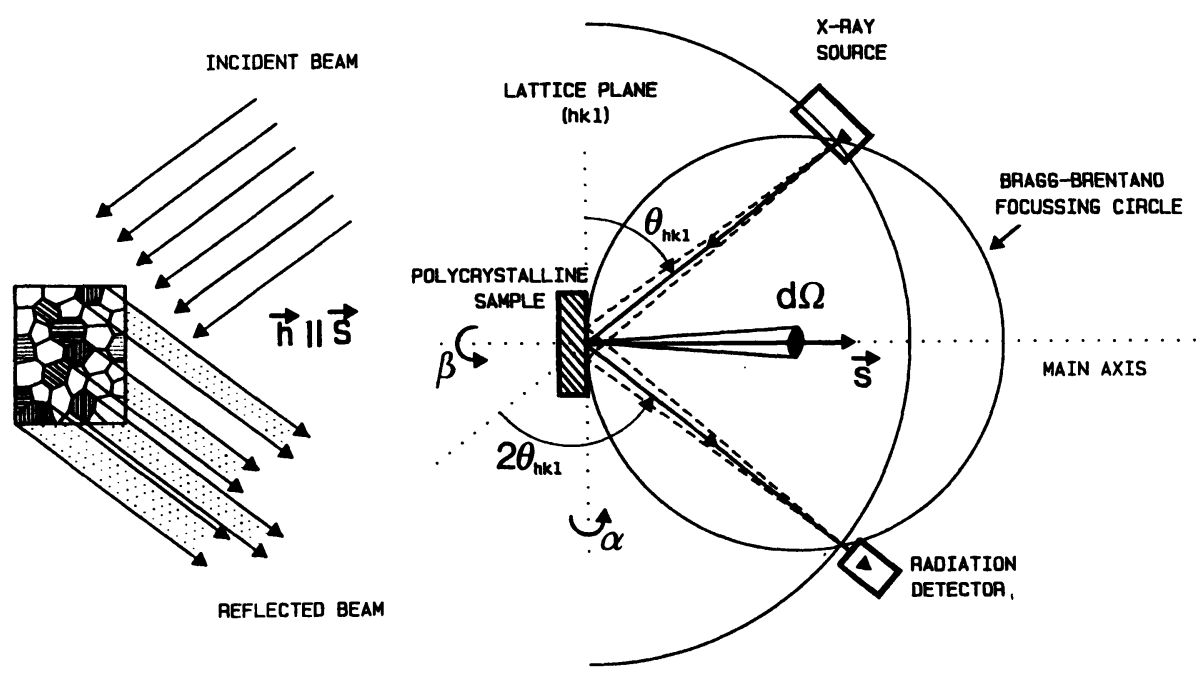

Figure 1 Physical principles of X-ray polycrystal diffraction. 
the ideal geometry, illustrated in Figure 2, caused among others by a finite extension of the focal spot in the X-ray tube, finite penetration depth in the sample material, finite spectral width of the used wavelength $\lambda$ and a finite irradiated area of the sample, parts of which are not on the diffraction circle, lead to peak defocalization.

As a consequence the diffracted intensity $\mathrm{I}_{(\mathrm{hkl})}(2 \theta)$ is distributed over an angular range $\Delta 2 \theta$ in the vicinity of the ideal focusing point given by the ideal Bragg angle $2 \theta_{\text {(hk) }}$. The intensity distribution over $2 \theta$ may be expressed in terms of the peak profile function $b_{(\mathrm{hkl})}(2 \theta)$ and the integral intensity $I_{(\text {hkl) }}^{\text {int }}$

$$
\mathrm{I}_{(\mathrm{hkl})}(2 \theta)=\mathrm{I}_{(\mathrm{hkl})}^{\mathrm{int}} \cdot \mathrm{b}_{(\mathrm{hkl})}(2 \theta)
$$

where the profile function is assumed to be normalized to unity

$$
\int_{2 \theta_{\mathrm{hkl}}-\Delta 2 \theta_{\max }}^{2 \theta_{\mathrm{hkl}}+\Delta 2 \theta_{\max }} \mathrm{b}_{(\mathrm{hkl})}(2 \theta) \cdot \mathrm{d}(2 \theta)=1
$$

The integration in Eq.(5) is to be extended up to a sufficient distance $2 \theta_{\max }$ from the ideal Bragg angle position $2 \theta_{\text {hkl }}$ covering the whole peak profile. The intensity $I_{(\text {hkl) }}(\alpha \beta)$ in Eq.(3) is then the integral intensity of a given reflection (hkl).

$$
\mathrm{I}_{(\mathrm{hkl})}(\alpha \beta)=\mathrm{I}_{(\mathrm{hkl})}^{\mathrm{int}}(\alpha \beta)
$$

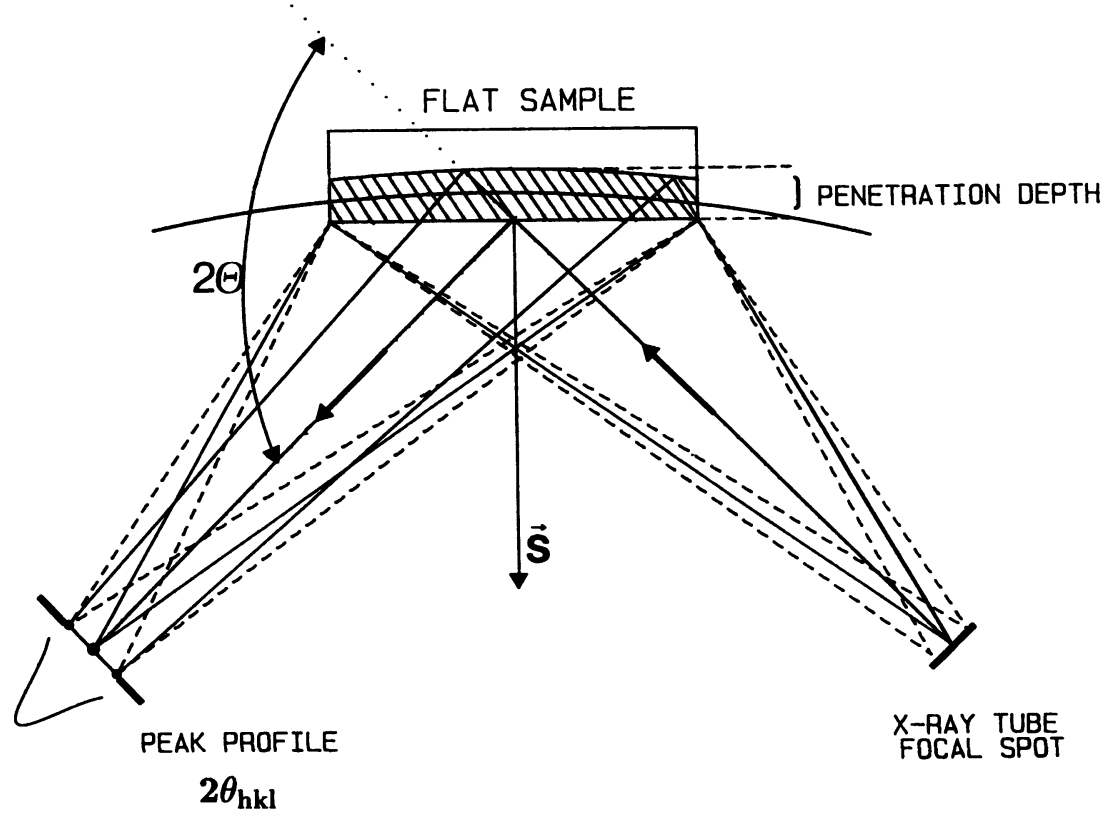

Figure 2 Deviations from the ideal Bragg-Brentano focusing geometry. 
The peak profile function $b_{(h k)}(2 \theta)$ may depend on several parameters, particularly, however on the Bragg angle and on the sample tilt angle.

\section{PRINCIPLES OF PSD TEXTURE MEASUREMENT}

A conventional texture diffractometer is equipped with a single detector usually a scintillation counter which is mounted on the $2 \theta$-circle. The single detector possesses a receiving slit which accepts incoming radiation within an angular range of $\Delta 2 \theta_{\text {slit }}$ about its central angle $2 \theta_{\mathrm{hkl}}$, see Figure 3 . Hence, the single detector measures an integral over the intensity $I_{(\mathrm{hkl})}^{\exp }$ limited only by the width of the used receiving slit.

$$
\mathrm{I}_{(\mathrm{hkl})}^{\exp }=\mathrm{I}_{(\mathrm{hkl})}^{\mathrm{int}} \cdot \int_{2 \theta_{\mathrm{hkl}}-\Delta 2 \theta_{\text {slit }}}^{2 \theta_{\mathrm{hkl}}+\Delta 2 \theta_{\text {slit }}} \mathrm{b}_{(\mathrm{hkl})}(2 \theta) \cdot \mathrm{d}(2 \theta)
$$

If the receiving slit is wide enough such that the whole profile of the diffraction line falls into it then the measured intensity is directly the integral intensity, Eq. (7). Usually

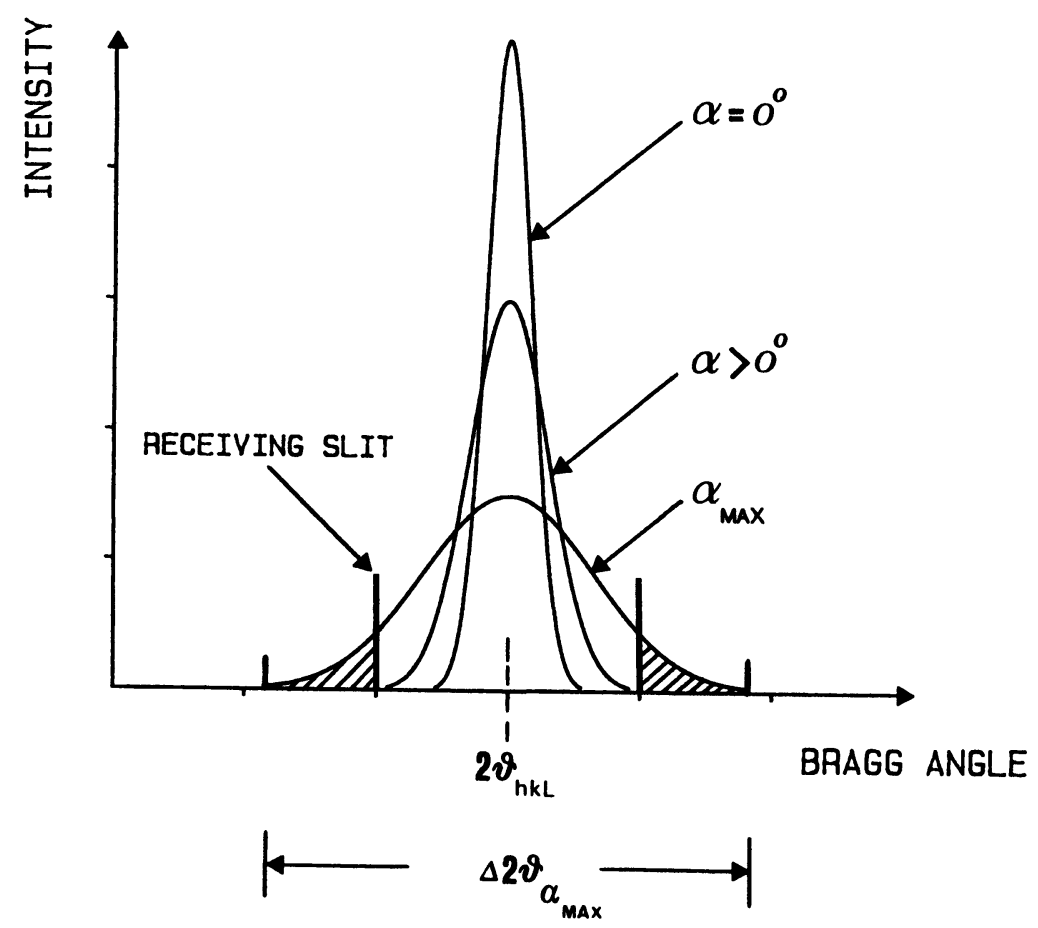

Figure 3 Peak profile cut-off effect with increasing peak width for a finite width of the receiving slit in the single detector method. 
this situation can be assumed at the normal sample position and at small to medium sample tilt angles. With increasing sample tilt angle, see Figure 3, the peak profile becomes broader and broader such that finally $\Delta 2 \theta_{\text {slit }}<\Delta 2 \theta_{\text {profile }}$. Then the integral in Eq.(7) is smaller than unity and the integral intensity can be obtained from the measured one only with an appropriate defocalization correction factor $\mathrm{D}_{(\mathrm{hkl})}(\alpha)$

$$
\mathrm{I}_{(\mathrm{hkl})}^{\exp }=\mathrm{I}_{(\mathrm{hkl})}^{\mathrm{int}} \cdot \mathrm{D}_{(\mathrm{hkl})}(\alpha)
$$

The defocalization corrections $D_{\text {(hkl) }}(\alpha)$ are mostly determined experimentally using a random specimen but they can also be computed. This method of the determination of integral intensities can only be applied if neighbouring diffraction lines in the spectrum are sufficiently separated such that no peak overlap occurs even at higher sample tilt angles.

The conventional, single detector-based, pole figure measuring method can not work, however, in complex line-rich diffraction spectra where abundant peak overlap takes place. In this case it is necessary to measure the complete diffraction spectrum in the required angular range $\Delta 2 \theta$ with a sufficient angular resolution $d(2 \theta)$ for each sample orientation and to apply an appropriate deconvolution procedure. This can also be accomplished with a single detector using sufficiently narrow detector slits (e.g. $0.1^{\circ}$ ) and scanning successively the whole diffraction profile. This is, however, a very timeconsuming method because the narrow slit receives only a very low intensity and the necessary measuring times become then unacceptably long. The only reasonable and practicable alternative to a single detector is then to use a position sensitive detector (PSD).

The PSD registers the diffracted intensities $\mathrm{I}(2 \theta)$ in a whole accessible angular range $\Delta 2 \theta$ with a preset angular resolution $\mathrm{d}(2 \theta)$ simultaneously. The available angular range is essentially broader than the width of the receiving slit used normally with a single detector. The PSD is mounted on the detector arm of a conventional texture diffractometer, Figure $4 \mathrm{a}$, with its center in the middle of the detector arm which

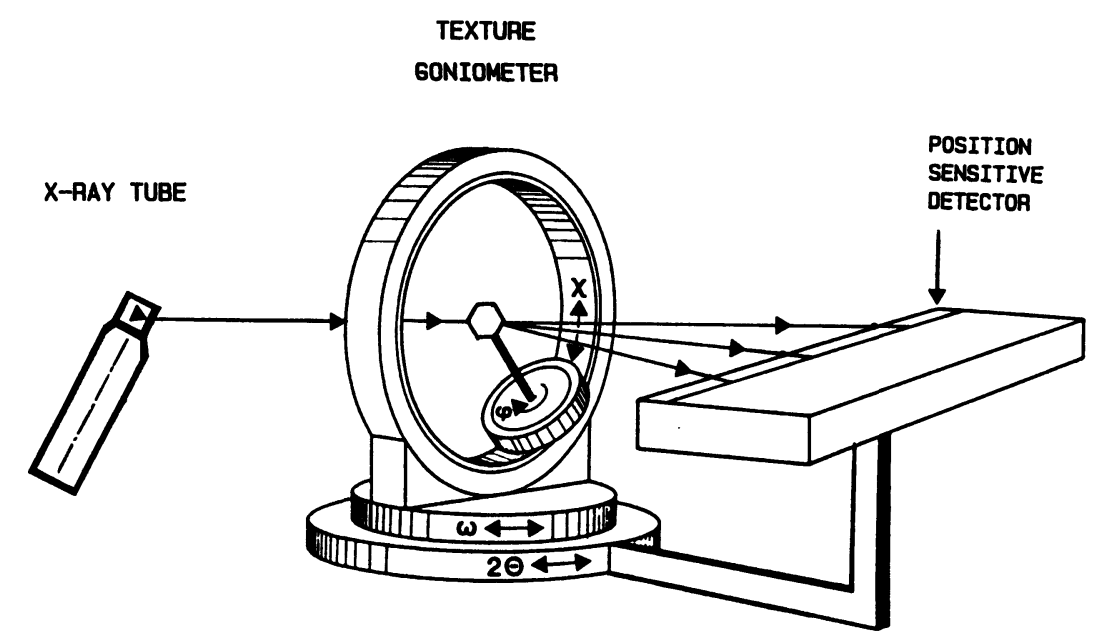

Figure 4a X-ray diffraction assembly with a four-circles texture goniometer and a position sensitive detector. 
corresponds to the actual angular position $2 \theta_{0}$. The detector electronics is able to distinguish the relative position $2 \theta_{\text {rel }}$ within the detector where a particular X-ray photon is detected and to count the corresponding pulses in the allocated channel of a multichannel analyser. Furthermore, the controlling software recognizes the actual $2 \theta_{0}$ position of the detector center such that each incoming X-ray pulse is counted in the channel corresponding to its actual $2 \theta$-position.

$$
2 \theta=2 \theta_{0}+2 \theta_{\text {rel }}
$$

The detector thus "sees" an angular interval $\Delta 2 \theta$ at the same time as is shown in Figure $4 \mathrm{~b}$. In the present diffractometer configuration the simultaneous and fully homogeneous "viewing angle" of the detector was approximately $\Delta 2 \theta=9^{\circ}$.

With a single detector method several pole figures are measured sequentially, one by one, with the focusing condition being strictly fulfilled for each particular Braggreflection (hkl). The detector location $2 \theta_{i}$ corresponds always to the peak maximum and the diffraction vector $\vec{S}_{\mathrm{i}}$ remains each time parallel to the sample normal direction in the zero position. Applying a position sensitive detector, the focusing condition can only be fulfilled for one position (one reflection) within the PSD and the non-focused diffraction peaks are thus slightly broadened. With the particular PSD used here this effect is, however, very small and can be neglected compared with the "natural" peak width resulting from the divergences of incident and reflected beam used in texture measurements (Wcislak and Bunge, 1991).

The application of the position sensitive measuring technique to texture analysis brings certain advantages compared to the single-detector method leading to a considerable improvement in measurement efficiency. The PSD-based measuring method is characterized by a simultaneous detection of many Bragg-reflections, registration of whole peak profiles in the diffraction spectrum instead of integral intensities only,

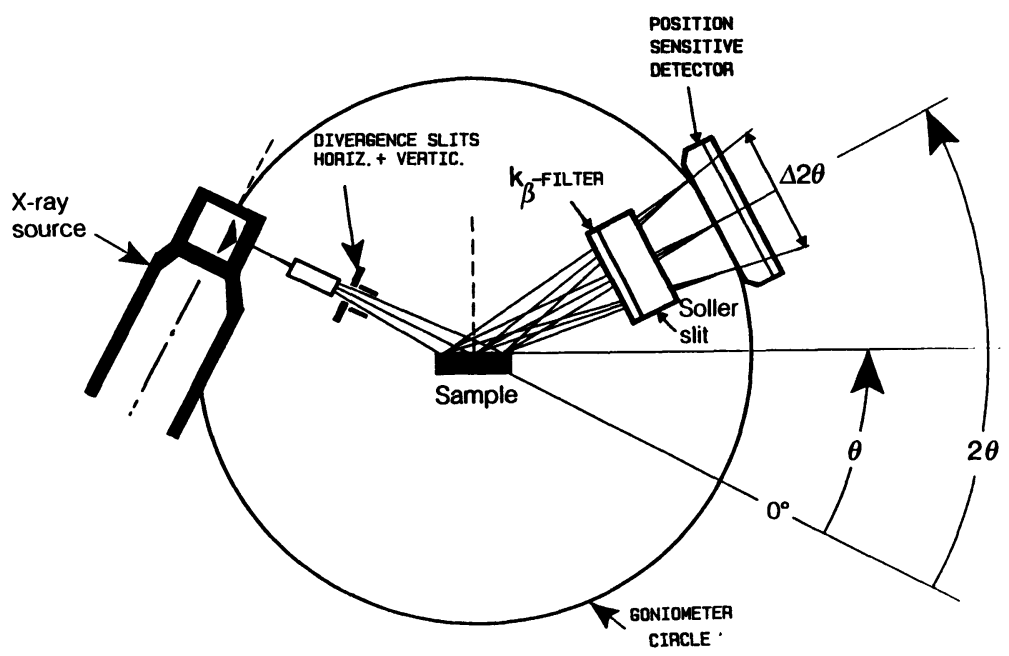

Figure $4 \mathrm{~b} \mathrm{X}$-ray diffraction measuring geometry for flat samples with the application of a linear position sensitive detector. 
increase of counting statistics and minimization of measuring error, decrease of global measuring time, simultaneous determination and reliable elimination of background scattering as well as elimination and compensation of measuring errors based on an insufficient device or sample adjustment.

\section{EVALUATION OF PSD DIFFRACTION SPECTRA}

The measurement of X-ray diffraction spectra based on the application of location sensitive detecting technique can be realized in various detector operating modes, i.e. in a stationary, stepwise stationary, and moving detector mode (Wcislak et al., 1992). The final result of each PSD run with any of these scanning modes is always a continuous $2 \theta$ diffraction spectrum with a preset angular resolution $\mathrm{d}(2 \theta)$ taken for a given sample orientation $\{\omega, \chi, \varphi\}$. Texture measurement with a position sensitive detector bases thus on the numerical evaluation of diffraction spectra $\mathrm{I}(2 \theta,\{\omega, \chi, \varphi\})$. The spectra have to be evaluated in two respects, namely the goniometer angles $\{\omega, \chi, \varphi\}$ have to be transformed into the pole figure angles $\{\alpha \beta\}$ and the spectrum over $2 \theta$ has to be evaluated for the required integral intensities $I_{(\mathrm{hkl})}^{\mathrm{int}}(\alpha \beta)$ (Wcislak et al., 1993). These two operations can be usually carried out independent of each other. Hence, it is needed only to consider the diffraction spectra $\mathrm{I}(2 \theta)$ (independent of $\{\omega, \chi, \varphi\}$ ) and to evaluate them for integral intensities.

When the different diffraction peaks are sufficiently separated from each other integral intensities can be obtained by simple integrating over the peak profile.

$$
I_{(\mathrm{hkl})}^{\mathrm{int}}=\int_{2 \theta_{\mathrm{hkl}}-\Delta 2 \theta_{\max }}^{2 \theta_{\mathrm{hkl}}+\Delta 2 \theta_{\max }} I_{(\mathrm{hkl})}(2 \theta) \cdot \mathrm{d}(2 \theta)
$$

This method is completely equivalent to the single detector method only that now the integration is carried out "mathematically" whereas in the single detector method it is done "physically" by the detector receiving slit.

In Eq. (4) the peak profile function $b_{(\mathrm{hkl})}(2 \theta)$ was not further specified. It may, however, be assumed that it can be expressed in a relatively simple mathematical form which depends only on a few parameters. Particular functions which can be used to describe diffraction profiles are the Gauss function $\mathrm{f}^{\mathrm{G}}$, the Cauchy function $\mathrm{f}^{\mathrm{C}}$ and the PseudoVoigt function $\mathrm{f}^{\mathrm{PV}}$ having the following forms (Parrish and Huang, 1980)

$$
\begin{aligned}
& \mathrm{b}^{\mathrm{G}}=\mathrm{f}^{\mathrm{G}}(\mathrm{x}, \sigma)=\mathrm{I}_{0} \cdot \exp \left(-\mathrm{k}_{\mathrm{G}} \mathrm{x}^{2}\right) ; \mathrm{k}_{\mathrm{G}}=\ln 2 /(\sigma / 2)^{2} \\
& \mathrm{~b}^{\mathrm{C}}=\mathrm{f}^{\mathrm{C}}(\mathrm{x}, \sigma)=\mathrm{I}_{0} /\left(1+\mathrm{k}_{\mathrm{C}} \mathrm{x}^{2}\right) ; \mathrm{k}_{\mathrm{C}}=1 /(\sigma / 2)^{2} \\
& \mathrm{~b}^{\mathrm{PV}}=\mathrm{f}^{\mathrm{PV}}(\eta)=\left[\eta \cdot \mathrm{f}^{\mathrm{G}}+(1-\eta) \cdot \mathrm{f}^{\mathrm{C}}\right] ; 0 \leq \eta \leq 1
\end{aligned}
$$

where the argument $\mathrm{x}$ is defined as the local distance from the centre of the peak at $2 \theta_{\mathrm{i}}, x=2 \theta-2 \theta_{\mathrm{i}}$ and the parameter $\sigma$ describes the full peak width at half maximum (FWHM). The Pseudo-Voigt function possesses a further shape factor $\eta$ which is defined as a weighting factor between the Gauss and Cauchy function.

With one of these functions the intensity distribution $\mathrm{I}_{\mathrm{i}}(2 \theta)$ of any diffraction peak in the spectrum can be modelled in the form

$$
\mathrm{I}_{\mathrm{i}}^{\text {model }}(2 \theta)=\mathrm{B}(2 \theta)+\mathrm{I}_{\mathrm{i}}^{\mathrm{int}} \cdot \mathrm{b}_{\mathrm{i}}^{\text {model }}(2 \theta)_{2 \theta_{1}, \sigma_{\mathrm{i}}}
$$


where $\mathrm{B}(2 \theta)$ is the background value in the vicinity of Bragg-angle $2 \theta_{\mathrm{i}^{-}}$. We have written the index $\mathrm{i}$ instead of (hkl) as in Eq. (4) thereby admitting that the peak in the spectrum may be composed of several (hkl) having the same Bragg-angle.

If the spectrum contains more than one peak at different central $2 \theta_{i}$ angles (consisting of one or several (hkl) each) then the model spectrum has the form

$$
I^{\text {model }}(2 \theta)=\mathrm{B}(2 \theta)+\sum_{\mathrm{i}} \mathrm{I}_{\mathrm{i}}^{\mathrm{int}} \cdot \mathrm{b}_{\mathrm{i}}^{\text {model }}(2 \theta)_{2 \theta_{1}, \sigma_{\mathrm{i}}}
$$

The model spectrum is fitted to the experimental one $I^{\exp }(2 \theta)$ according to the least squares condition.

$$
\int_{2 \theta_{\min }}^{2 \theta_{\max }}\left[\mathrm{I}^{\exp }(2 \theta)-\mathrm{I}^{\text {model }}(2 \theta)\right]^{2} \cdot \mathrm{d}(2 \theta)=\min \left(\mathrm{I}_{\mathrm{i}}, 2 \theta_{\mathrm{i}}, \sigma_{\mathrm{i}}\right)
$$

The minimum is taken with respect to the peak profile parameters i.e. the peak positions $2 \theta_{\mathrm{i}}$, the peak heights $I_{\mathrm{i}}$ and the peak half-widths $\sigma_{\mathrm{i}}$. The use of the index $\mathrm{i}$ for a certain peak rather then (hkl) emphasizes once more, that it is not altogether necessary to know the indices (hkl) in order to solve the above equation. One peak i may consist of several (hkl) and even, in the case of multiphase spectra, it may contain peaks of several phases.

In Eq.(14) the minimum is assumed under the variability of all parameters of the model function. It may, however, be meaningful to keep some of the parameters fixed as far as they are known beforehand. The following situations are to be considered:

\section{Unknown Diffraction Spectrum}

The peak positions and their half-widths in the spectrum are not exactly known. In this case all profile parameters, i.e. $\mathrm{B}(2 \theta), \mathrm{I}_{\mathrm{i}}, 2 \theta_{\mathrm{i}}, \sigma_{\mathrm{i}}$ are variable in Eq.(14). This situation can be encountered when texture analysis of an unknown material is started. Usually it will be necessary to know the crystal structures of the phases in the material. Hence, the peak sequence $2 \theta_{i}$ in the spectrum should be known in principle. But it may be that the values $2 \theta_{i}$ are not exact enough to rely on them absolutely. The same may be assumed in this case for the half-widths $\sigma_{i}$ of the peaks.

\section{Texture Analysis}

After the preliminary measurements with a new material, it may be assumed that the peak positions $2 \theta_{i}$ are known. If the texture goniometer is well adjusted then the $2 \theta_{i}$ values should be independent of the Eulerian cradle angles $\{\omega, \chi, \varphi\}$ The individual spectra for individual sample angles can then be analysed by minimizing Eq. (14) under the assumption:

$-2 \theta_{\mathrm{i}}$ are known and constant for all involved reflections,

$-\mathrm{B}(2 \theta), \mathrm{I}_{\mathrm{i}}, \sigma_{\mathrm{i}}$ are variable.

\section{Reduced Texture Analysis}

Finally it may be assumed that the half-widths $\sigma_{\mathrm{i}}$ depend only on the sample tilt angle i.e. are known functions of the pole figure angle $\alpha$. In this case it can be put: 
- $2 \theta_{\mathrm{i}}=$ const, $\sigma_{\mathrm{i}}=\sigma_{\mathrm{i}}(\alpha)$, and only the remaining parameters

$-B(2 \theta), I_{i}$ are variable.

This case may be assumed to be applicable in routine texture analysis.

In XRD texture analysis usually no monochromators are used. Rather, only a $\mathbf{k}_{\boldsymbol{\beta}}$ filter is being applied. Hence, a certain amount of white radiation with some intensity of $\mathrm{k}_{\beta}$-radiation is still present in the primary beam. This spectrum is "Bragg-reflected" at all involved lattice planes considering their reflectivity, pole density, absorption and other intensity factors. This constitutes one part of the background of the diffraction spectrum. Other parts of the background may be due to sample fluorescence, air scattering of X-rays, diffuse scattering in the sample and other contributions.

\section{PEAK PROFILE FITTING PROCEDURE}

As was already mentioned the diffraction peak profile depends on many variables such as intensity distribution in the focal spot of the X-ray tube, all divergences of the diffractometer, the penetration depth in the sample and others. From a practical point of view it is sufficient to determine the peak profile experimentally. For the separation of overlapping peaks the best fitting shape must be known and peak width should be expressed in terms of only one free parameter. For this purpose, three types of profile functions for symmetrical reflections, i.e. the above defined Gauss, Cauchy and PseudoVoigt functions, were tested.

The angular intervals within a diffraction spectrum fixed for the fitting procedure are relatively narrow (a range of a few degrees is a typical one), so that the provided graphic documentation of the fitting procedure displays normally a lot of profile details, see Figure 5. This figure illustrates the fitting process of a single, well-separated peak in the diffraction spectrum. The (111)-reflection taken from a random copper sample in the back-reflection method and PSD stationary mode was processed. The crosses represent the measured intensity values interpolated by the fitting curve. The distribution of the peak profile itself, free of background, as well as the difference curve with the discrepancy area is also depicted. This latter function illustrates graphicly the effectiveness of the fitting procedure. The relative fitting error (FE) is defined by Eq.(15) using the absolute values of the intensity deviation.

$$
\mathrm{FE}[\%]=\frac{\int_{2 \theta_{\min }}^{2 \theta_{\max }}\left|\mathrm{I}^{\exp }(2 \theta)-\mathrm{I}^{\mathrm{mod}}(2 \theta)\right| \cdot \mathrm{d}(2 \theta)}{\int_{2 \theta_{\min }}^{2 \theta_{\max }}\left|\mathrm{I}^{\exp }(2 \theta)-\mathrm{I}^{\mathrm{bac}}(2 \theta)\right| \cdot \mathrm{d}(2 \theta)} \cdot 100
$$

where $\mathrm{I}^{\mathrm{exp}}$ are the experimentally determined intensities, $\mathrm{I}^{\mathrm{mod}}$ are the theoretical intensities calculated from the model functions, $I^{\text {bac }}$ are the local background values and the term $\mathrm{d}(2 \theta)$ is an integration element corresponding to the detection resolving power. The value of the fitting error depends, of course, on the chosen fitting interval $\left(2 \theta_{\max }-\right.$ $\left.2 \theta_{\min }\right)$ and fitting error values must thus be compared only within identical integration intervals. The usefulness of several fitting functions in the profile analysis for raw 


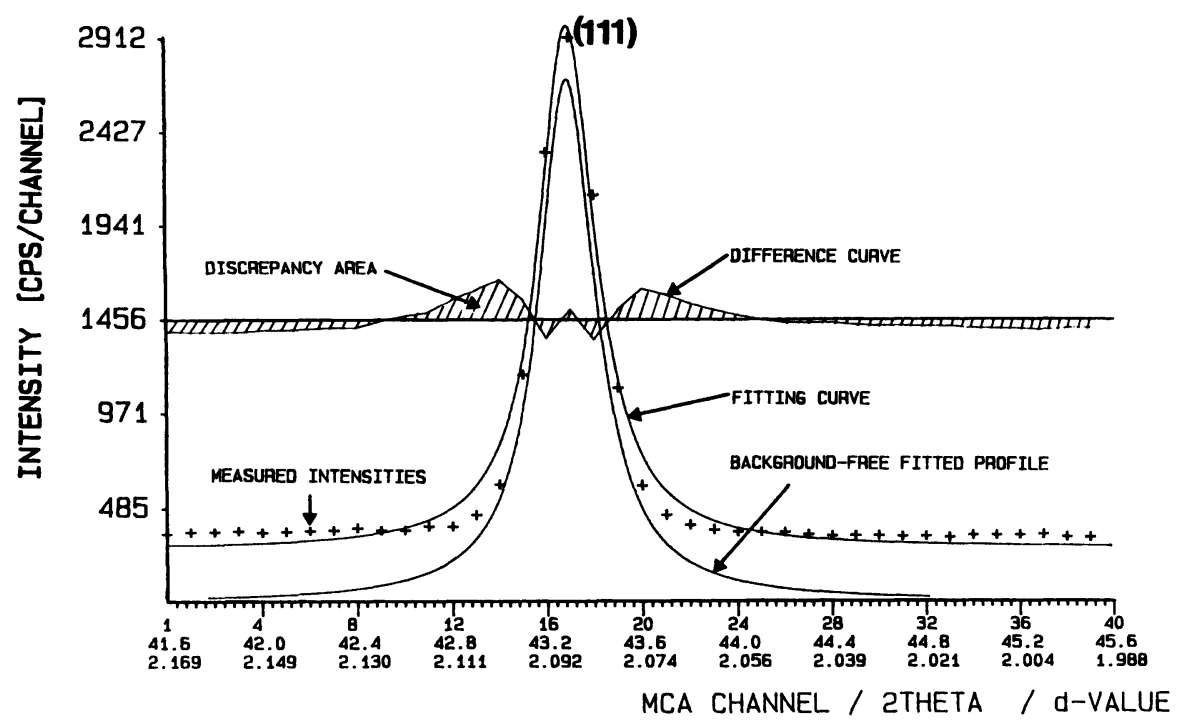

Figure 5 Graphic documentation of the profile fitting procedure displaying several profile details.

diffraction data obtained with the PSD/MCA method was carefully examined. Various samples (random and strongly textured) from materials with different crystals structures and for different (hkl)-reflections lying in the low, medium and high $2 \theta$-ranges were subject to analysis to find out the best fitting curve. It was found that:

- The profile fitting efficiency is independent of the data acquisition way (data obtained with the PSD working in stationary or moving detector mode).

- Independently of the $2 \theta$-range the Gauss curve $\mathrm{f}^{\mathrm{G}}$ gives the best fitting results (the lowest fitting errors) compared to the Cauchy $\mathrm{f}^{\mathrm{C}}$ and standard Pseudo-Voigt $\mathrm{f}^{\mathrm{PV}}$ $(\eta=0.5)$ fitting functions, see details in Figure 6. Profile fitting procedures using different model functions were performed on the same data set of one well-separated (111)-reflection taken from a random copper powder sample.

- Further considerable improvement in the profile fitting accuracy is achieved by the activation of the weighting factor $\eta$ in the Pseudo-Voigt fitting function and the consideration of the $\mathbf{k}_{\alpha_{2}}$ radiation component. The positive influence of these factors on the fitting procedure accuracy is illustrated in Figure 7 for some (hkl)-reflections representative for low, medium and high Bragg angles, respectively. Profile fitting procedures were carried out on particular well-separated reflections, (111), (222) and (420) respectively, taken in the PSD stationary mode from a random copper powder sample.

- The influence of $\eta$ in the Pseudo-Voigt function on the fitting accuracy is especially distinct at low Bragg angles, see Figure 7a, bringing an improvement of nearly $100 \%$ (the relative fitting error decreases almost by factor 2$)$ for $\mathrm{f}^{\mathrm{PV}}(\eta=0.85)$. The positive influence of $\eta$ on the fitting efficiency for medium Bragg angles is weaker, but it is still nearly $25 \%$, see Figure $7 \mathrm{~b}$. 


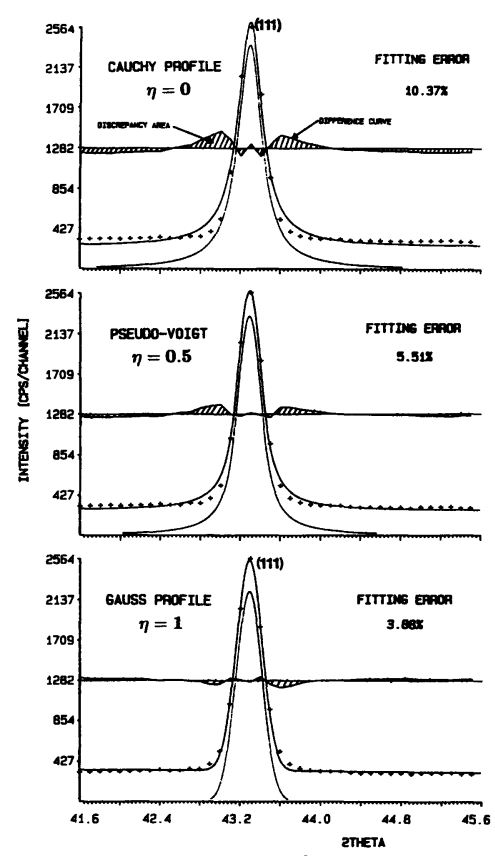

Figure 6 Peak profile fitting effectivity using different standard model functions on the same data set taken from a random copper sample.

The influence of the $\mathrm{k}_{\alpha 2}$-component on the fitting accuracy for low and medium Bragg angles is rather weak and can be, in many cases, simply neglected, see Figure $7 \mathrm{a}$ and Figure $7 \mathrm{~b}$. On the other hand its influence on the fitting efficiency for high Bragg angles is tremendous (accuracy improvement by several hundred percent), see Figure 7c. In other words, a reliable effective profile fitting without the consideration of the $\mathbf{k}_{\alpha 2}$-component is, for higher Bragg angles, practically impossible.

The results of the above tests of profile fitting effectiveness are summarized in Figure 8 which shows the fitting error values as a function of Bragg angles without and with the consideration of the weighting factor $\eta$ and the $\mathbf{k}_{\infty 2}$ radiation component. It is clearly seen that the consideration of the weighting factor significantly improves the fitting accuracy for low Bragg angles and the consideration of the $\mathbf{k}_{\alpha 2}$ component extends the possibility of effective profile analysis for higher Bragg angles. Hence, the Pseudo-Voigt function with an optimum weighting factor and the consideration of the line doublet should be used as the best fitting procedure.

From the point of view of pole figure measurement based on the integral intensities of peaks it is also very important to know, how the peak fitting quality depends on the sample tilt angle (increasing defocalization and peak broadening). It was found that each fitting function works the better the broader the peak profiles are. This is clearly 
seen in Figure 9 showing the fitting procedure for the (111)-reflection of a random copper sample taken at three different sample tilt angles. The Pseudo-Voigt function with the consideration of optimum weighting factor and line doublet was applied. One sees that the diffraction line is excellently fitted with decreasing fitting error at increasing sample tilt. Due to increasing defocalization effects with increasing sample tilt the peak maximum intensity decreases and simultaneously the peak width increases. On the other hand, the integral intensity (peak area) remains practically constant within the measuring error of $\pm 3 \%$. This means that the PSD-based pole figure measurement with the employment of profile analysis can be successfully realized without the involvement of time-consuming and faulty defocalization corrections (Wcislak et al., 1993).

The optimum profile parameters of several peaks from Figure 9 (three exemplary sample tilts) provided by the profile fitting procedure based on the optimized PseudoVoigt function are put together in Table 1.

\section{DIFFRACTION PROFILE POLE FIGURES}

Permitting the variability of all profile parameters of an individual (hkl)-reflection during the iterative minimization of Eq.(14), the profile fitting procedure provides their optimum values. The following peak profile parameters are thus obtained; the peak intensity $I_{(h k l)}$, the peak position $2 \theta_{(h k l)}$, the peak half-width $\sigma_{(\mathrm{hkl})}$ as well as the most reliable local background level $B_{(\text {(hkl) }}$ in the vicinity of Bragg-reflection (hkl). Having the optimum peak profile parameters and knowing the analytical form of fitting function the most accurate value of integral intensity (peak area) $\mathrm{II}_{(\mathrm{hkl})}$ can be calculated. The effectiveness of each profile fitting procedure is then characterized by the minimum fitting error $\mathrm{FE}_{(\mathrm{hkl})}^{\min }$ according to Eq.(15).

The availability of all these optimized parameters of a given diffraction profile in each sample orientation $\{\omega, \chi, \varphi\}$ allows to define and construct new generalized diffraction profile "pole figures". Besides the conventional integral intensity pole figures (IIPF) the following new kinds of pole figures can be considered; peak maximum intensity pole figures (PIPE), peak position pole figures (PPPF), peak width pole figures (PWPF), background level pole figures (BLPF) and fitting error pole figures (FEPF) (peak position pole figures correspond to lattice strain pole figures, see e.g. Hoffmann et al. 1986).

Table 1 Optimum peak profile parameters of a random sample provided by the profile fitting procedure

\begin{tabular}{lccc}
\hline (111)-reflection & $\alpha=0^{\circ}$ & $\alpha=40^{\circ}$ & $\alpha=70^{\circ}$ \\
\hline Peak intensity & 12309 & 5575 & 2902 \\
Peak position & $43.29^{\circ}$ & $43.28^{\circ}$ & $43.27^{\circ}$ \\
Peak FWHM & $0.31^{\circ}$ & $0.72^{\circ}$ & $1.39^{\circ}$ \\
Peak area & 43584 & 43705 & 42985 \\
Background level & 1275 & 1284 & 1265 \\
Fitting error & $1.66 \%$ & $1.13 \%$ & $1.01 \%$ \\
\hline
\end{tabular}




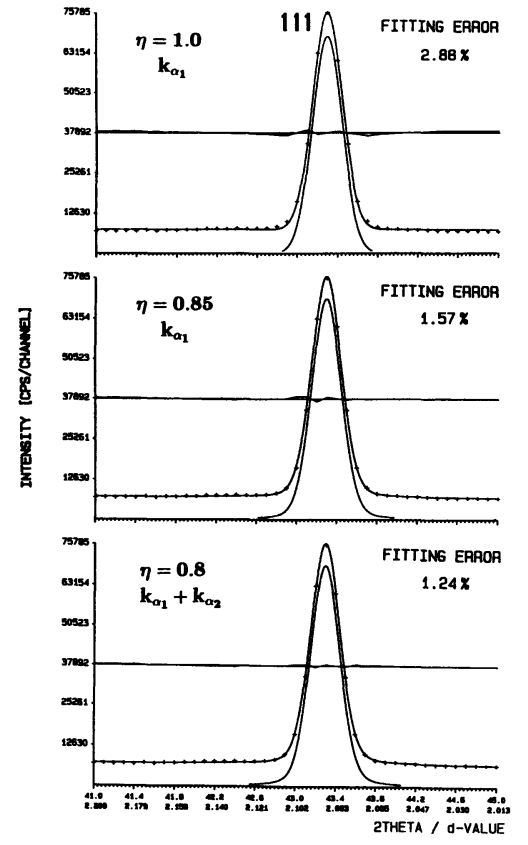

(a)

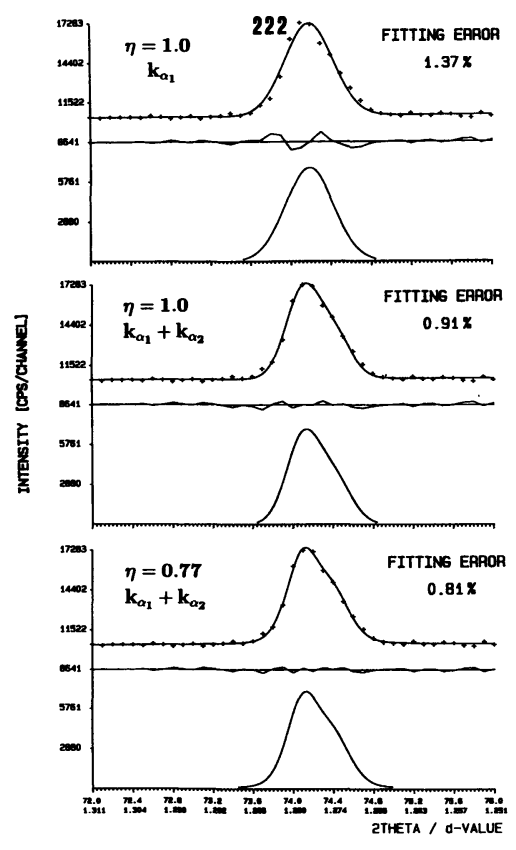

(b)

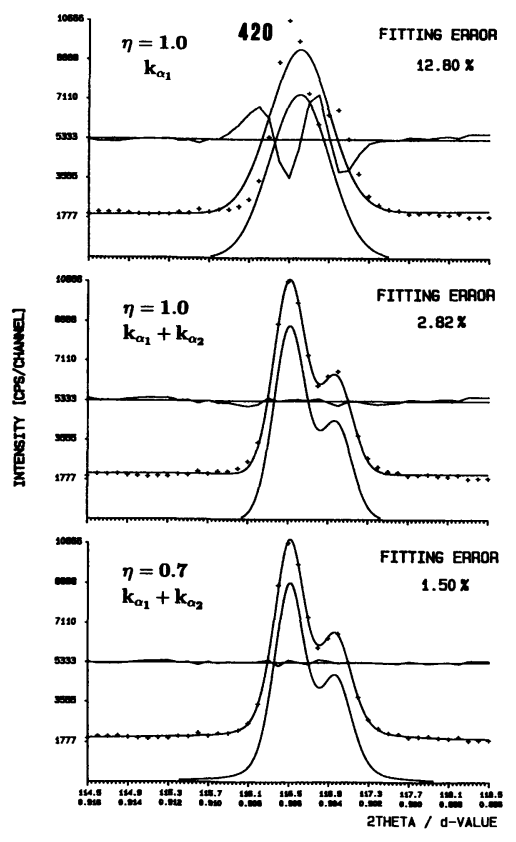

(c)

Figure 7 Peak profile fitting using the Pseudo-Voigt function with the consideration of the weighting factor $\eta$ and the line doublet $\alpha_{1} / \alpha_{2}$ for a random copper sample

- a) low Bragg angle (111)-reflection.

- b) medium Bragg angle (222)-reflection.

- c) high Bragg angle (420)-reflection. 


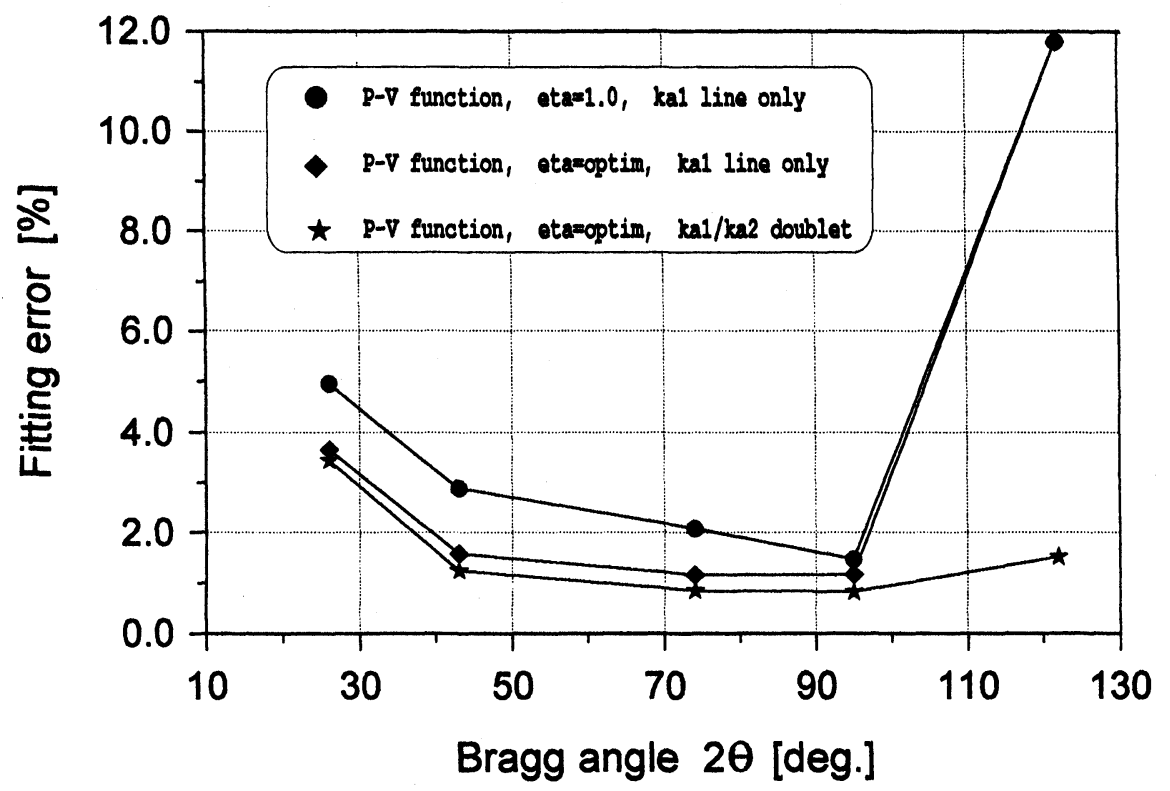

Figure 8 Profile fitting accuracy as a function of Bragg angle without and with the consideration of the weighting factor $\eta$ and the line doublet $\alpha_{1} / \alpha_{2}$.

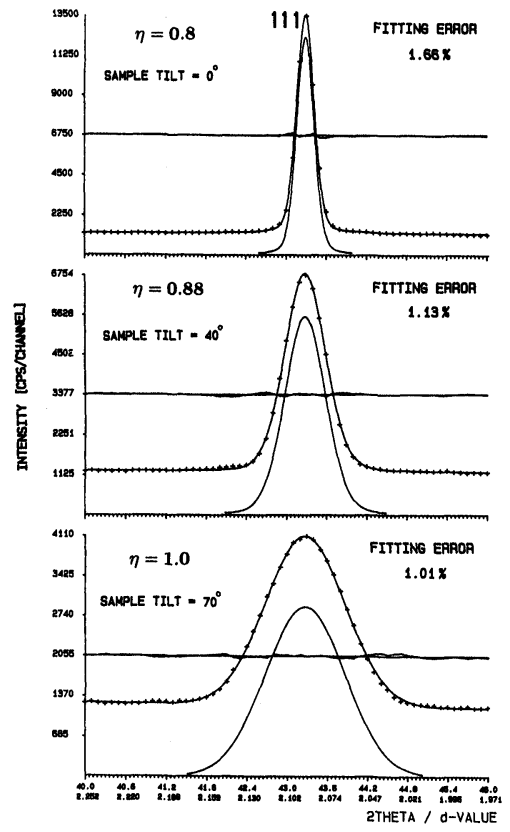

Figure 9 Pseudo-Voigt fitting procedure for the (111)-reflection of a random copper sample taken at three different sample tilt angles. 
The applicability of the PSD measuring technique and the effectiveness of the peak profile analysis for the determination of diffraction profile pole figures was tested with the well-known rolling texture of copper. The PSD was used "conventionally" i.e. it was controlled in such a way that its center was always located exactly in the (hkl)reflection position. With these conditions no coordinate transformations between the goniometer angles and pole figure angles were needed. The diffraction spectrum containing the (111)-reflection of a 95\% cold-rolled copper sheet was measured. The sample was taken from a rolled sheet of $2 \mathrm{~mm}$ thickness, the middle plane of which was analysed.

The required diffraction spectra were measured in the PSD stationary mode using the normal pole figure scanning schedule of $\Delta \alpha=5^{\circ}$ and $\Delta \beta=5^{\circ}$ up to the sample tilt of $\alpha_{\max }=70^{\circ}$. In each diffraction spectrum taken in the sample position $\{\alpha, \beta\}$ the profile fitting procedure was carried out providing the optimum peak profile parameters. The fitting procedure used was based on the Pseudo-Voigt function taking into account the line doublet and applying the optimum weighting factor $\eta$ bringing the lowest fitting error.

All peak profile parameters can then be plotted as diffraction profile pole figures. The results are presented in Figure 10 and Figure 11 for the (111) -reflection expressed in absolute values of a given quantity without any normalization procedure. The diffraction profile pole figures are demonstrated either in the normally applied iso-line presentation or in the iso-colour (grey colours scale) presentation. The iso-line presentation provides much more details and can be easier interpreted quantitatively. On the other hand, the iso-colour presentation gives a better image about the position

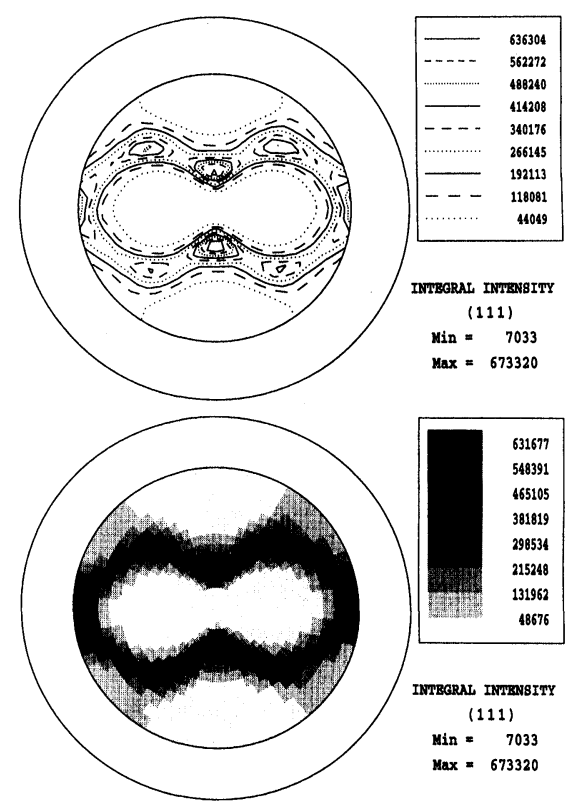

Figure 10 The conventional integral intensity pole figure of the (111)-reflection of a cold-rolled copper sheet shown both in the iso-line and iso-colour presentation expressed in absolute values without normalization procedure. 


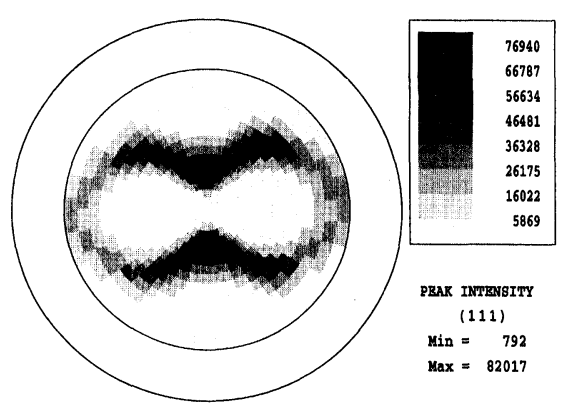

(a)

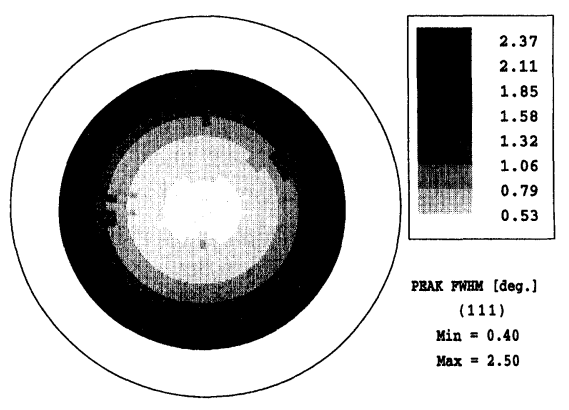

(c)

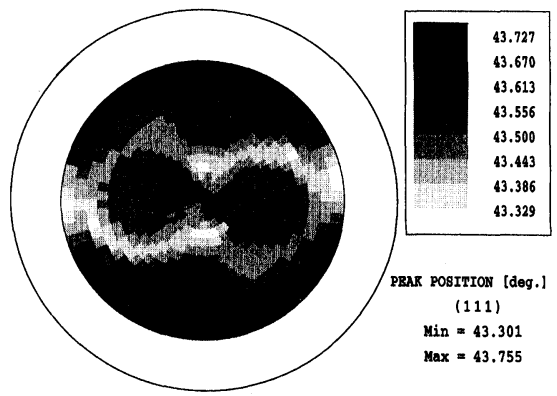

(b)

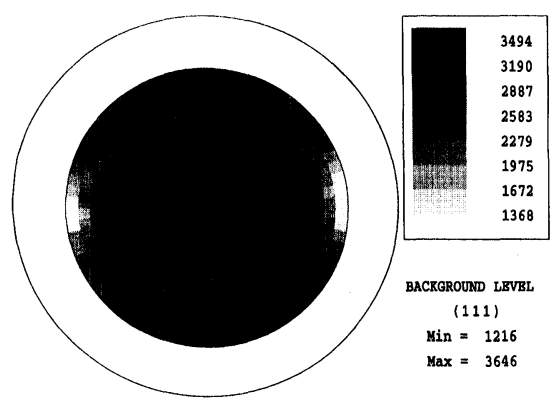

(d)

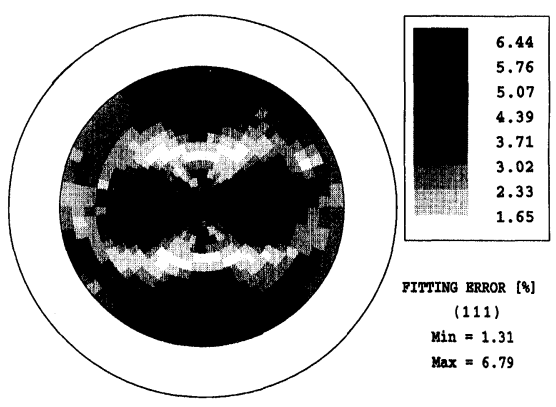

(e)

Figure 11 Generalized diffraction profile pole figures of the copper sheet of Figure 10, shown in the iso-colour presentation expressed in absolute values

- a) peak maximum intensity.

- b) peak position.

- c) peak width.

- d) background level.

- e) fitting error. 
of local maxima and minima within each pole figure. Figure 10 shows the conventional integral intensity pole figure (both in the iso-line and iso-colour presentation) containing the texture information of the sample. Figure 11a to Figure 11e illustrate the peak maximum intensity, peak position, peak width, background level and fitting error pole figures, respectively, in the iso-colour presentation.

In an other experiment the determination of diffraction profile pole figures was tested with a polymer sample. Texture analysis in polymers is significantly more complicated as compared to metallic materials. Whereas metals are completely crystalline, polymers may contain considerable amounts of non-crystalline structure. This leads to relatively weak diffracted intensities and low peak-to-background ratios. With lower crystal symmetries and higher lattice parameters the polymers are also characterized by more complicated peak-rich diffraction spectra.

A $0.55 \mathrm{~mm}$ thick polyethylene sample with orthorhombic crystal symmetry was used in this test. The measurements of diffraction spectra were performed in the PSD moving mode and back-reflection technique. Figure 12 shows the diffraction spectrum taken in the normal zero-tilt sample position in the whole relevant angular range. The PSD was then moved in the $2 \theta$-range of $\left(15^{\circ}-30^{\circ}\right)$ with the constant angular velocity of $30 \%$ min. This part of the diffraction spectrum contains two close-lying but not overlapped reflections (110) and (200). The diffraction spectra containing the investigated pair of reflections were measured using the normal pole figure scanning schedule of $\Delta \alpha=5^{\circ}$ and $\Delta \beta=5^{\circ}$ up to $\alpha_{\max }=70^{\circ}$. In each diffraction spectrum, taken in the appropriate sample position, the profile fitting procedure was performed to obtain the optimum profile parameters of both reflections. The applied fitting procedure was based again on the Pseudo-Voigt function considering the line doublet and optimum weighting factor. The fitting procedure was performed in one fitting interval containing two separate reflections (110) and (200). This results in the optimum peak profile parameters related with both reflections separately and the optimum values of background and fitting error characteristic for the common fitting interval. The peak profile parameters were then plotted in the form of pole figures.

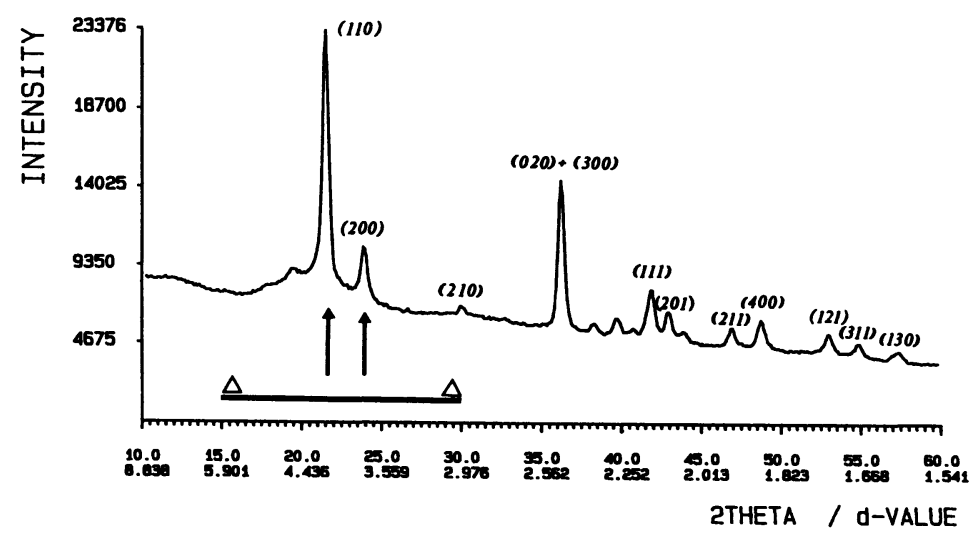

Figure 12 Diffraction spectrum of a polyethylene sample taken in the PSD moving mode with the scanning speed of $30 \% \mathrm{~min}$ in the normal zero-tilt sample position. 
The results are demonstrated in the iso-line and iso-colour presentation respectively with contour levels expressed in absolute values without normalization. Figure 13 shows the conventional integral intensity pole figures of both reflections (110) and (200). Next figures $14 \mathrm{a}$ and $14 \mathrm{~b}$ illustrate the peak position and peak width pole figures, respectively also for both reflections (110) and (200). Finally Figure 15 shows the background level and fitting error pole figures related with the fitting interval common for both reflections (110) and (200).

The conventional integral intensity pole figures contain the texture information of the specimen. The new "generation" of pole figures based on the optimum diffraction profile parameters contain also information about different other microstructural quantities. Particularly, the peak width pole figures are related to particle size studies, the peak position pole figures to lattice strain investigations and the background level pole figures to the analysis of amorphous phases or diffuse scattering. Besides that also different instrumental factors can be estimated and better separated from the sample effects. For instance, peak shift pole figures can be considered as a test of mechanical adjustment of the diffractometer and precision of sample preparation. Hence, a further evaluation of the obtained diffraction profile pole figures is necessary. This is particularly obvious in the peak-width pole figure, Figure 11c, which contains the greatest "instrumental part" due to sample tilt. In general the separation of "instrumental" and "physical" parts in the peak profile pole figures is not trivial. But this will not be considered in the present paper. Fitting error pole figures can be interpreted as a measure of global effectivity of the applied fitting procedure (raw data quality, kind of fitting function, number of admitted iteration steps and others). The conventional integral

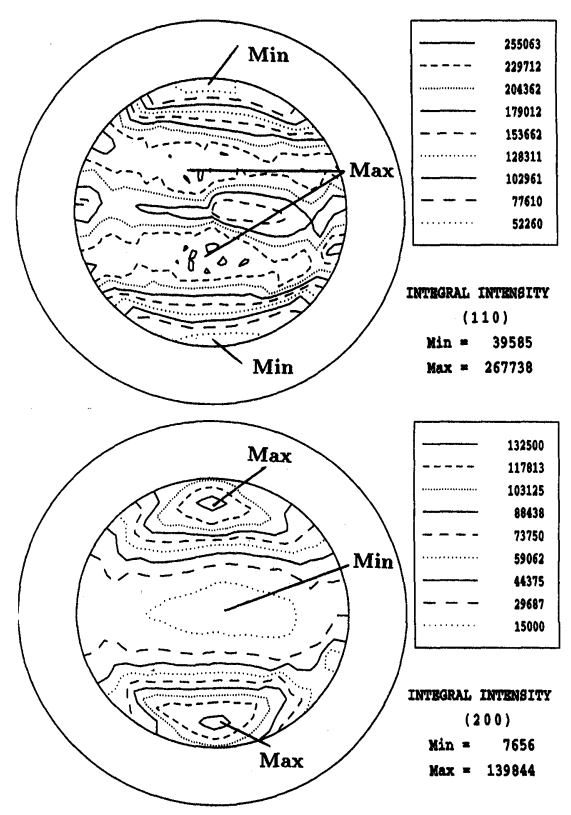

Figure 13 The conventional integral intensity pole figures of both reflections (110) and (200) of a polyethylene sample in the iso-line presentation expressed in absolute values. 


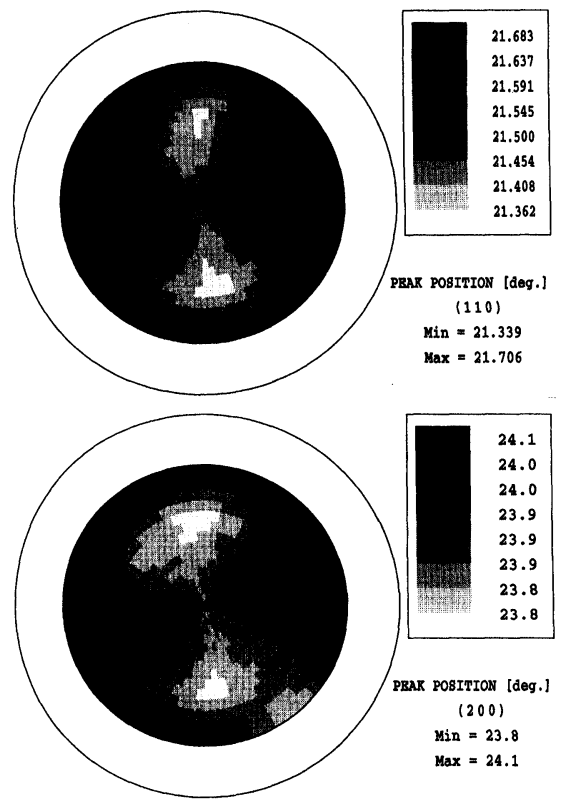

(a)

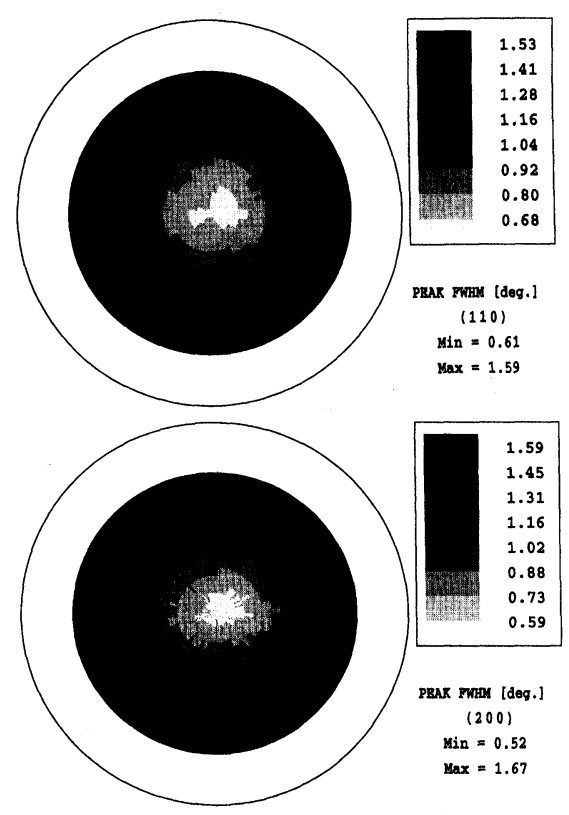

(b)

Figure 14 Generalized diffraction profile pole figures of the polyethylene sample of Figure 13, shown in iso-colour presentation expressed in absolute values

- a) peak position..

- b) peak width.

intensity pole figures can then be "tagged" with the fitting error as a "weighting factor" for ODF calculation.

\section{CONCLUSIONS}

An advanced measuring-analysing method for the determination of new generalized pole figures based on the diffraction profile parameters was developed. The usage of a position sensitive detector for diffraction measurements enables a simultaneous registration of some part of the spectrum and a discrete data assignment in the multichannel analyser. The availability of the complete diffraction pattern allows the profile analysis in the spectrum both on overlapped (profile deconvolution) and separated (profile fitting) reflections. The peak profile analysis provides the optimized peak profile parameters characterizing an individual (hkl) reflection. All these peak profile parameters are available in each sample position allowing the construction of generalized diffraction profile pole figures. These are the peak maximum intensity, peak position, peak width, local background and fitting error pole figures as well as the normally used integral intensity pole figures. These new pole figures contain valuable information about different microstructural quantities of the material. It may be forseen that they will 


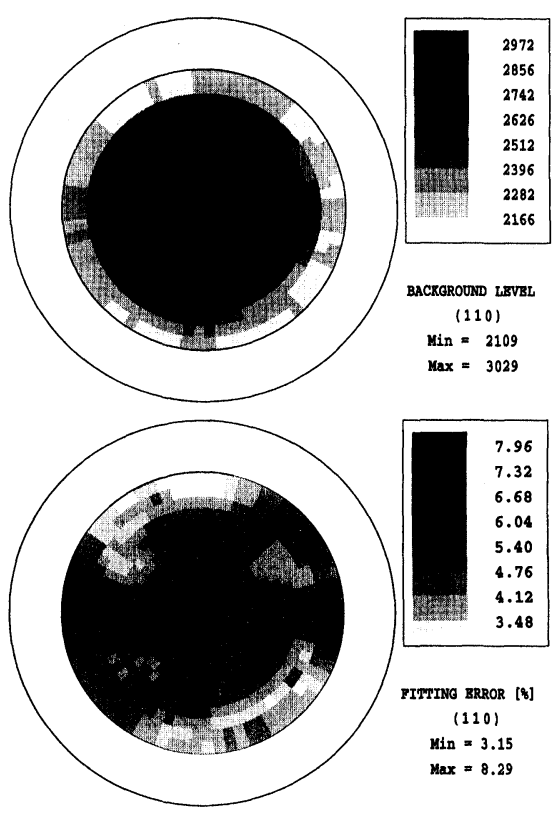

Figure 15 The background level and fitting error pole figures related with the fitting interval common for both reflections (110) and (200) in the iso-colour presentation expressed in absolute values.

find various applications in different fields of X-ray diffraction analysis as a competent investigation tool.

\section{Acknowledgement}

The authors are indebted to Dr. Yu. Perlovich for many stimulating discussions on the topic of peak profile analysis. We also gratefully acknowledge Mr. R. Hedel for making his graphics software available.

\section{References}

Bunge, H. J. (1982). Texture Analysis in Materials Science, Butterworth Publication, London.

Bunge, H. J. (Ed.) (1986). Experimental Techniques of Texture Analysis p. 1-28, DGM Informationsgesellschaft, Oberursel.

Bunge, H. J. and Puch, K. H. (1984). Principles of Texture Goniometer Measurements. Zeitschrift für Metallkunde, 75, 124-132.

Hoffmann, J., Maurer, G., Neff, H. and Macherauch, E. (1986). A PSD Diffractometer for the Determination of Texture and Lattice Deformation Pole Figures. In: Experimental Techniques of Texture Analysis. (Ed.) H. J. Bunge. DGM Informationsgesellschaft, Oberursel p. 409-418.

Parrish, W. and Huang, A. (1980). Accuracy of the Profile Fitting Method for X-ray Polycrystalline Diffractometry. NBS Special Publication, 567, 95-111.

Wcislak, L. and Bunge, H. J. (1991). Simultaneous Measurement of Pole Figures Using a Position Sensitive Detector. Proc. ICOTOM-9(1990), Textures and Microstructures, 14-18, 220-232.

Wcislak, L., Bunge, H. J., Haase, M. and Nauer-Gerhardt, C. (1992). X-ray Diffraction Texture Measurements Using a Linear PSD. Advances in X-ray Analysis. Plenum Press, 35, 309-319.

Wcislak, L., Bunge, H. J. and Nauer-Gerhardt, C. (1993). X-ray Diffraction Texture Analysis with a Position Sensitive Detector. Zeitschrift für Metallkunde, 84, 479-493. 